\title{
Target network differences between western drugs and Chinese herbal ingredients in treating cardiovascular disease
}

\author{
Peng Fu ${ }^{1,2}$, Linlin Yang ${ }^{3}$, Yi Sun ${ }^{3}$, Li Ye ${ }^{1}$, Zhiwei Cao ${ }^{1,2,3^{*}}$, Kailin Tang ${ }^{2,3^{*}}$ \\ From The 3rd ISV Pre-conference Computational Vaccinology Workshop (ICoVax 2013) \\ Barcelona, Spain. 26 October 2013
}

\begin{abstract}
Background: Western drugs have achieved great successes in CVDs treatment. However, they may lead to some side effects and drug resistance. On the other hand, more and more studies found that Traditional Chinese herbs have efficient therapeutic effects for CVDs, while their therapeutic mechanism is still not very clear. It may be a good view towards molecules, targets and network to decipher whether difference exists between anti-CVD western drugs and Chinese herbal ingredients.

Results: Anti-CVD western drugs and Chinese herbal ingredients, as well as their targets were thoroughly collected in this work. The similarities and the differences between the herbal ingredients and the western drugs were deeply explored based on three target-based perspectives including biochemical property, regulated pathway and disease network. The biological function of herbal ingredients' targets is more complex than that of the western drugs' targets. The signal transduction and immune system associated signaling pathways, apoptosis associated pathways may be the most important pathway for herbal ingredients, however the western drugs incline to regulate vascular smooth muscle contraction associated pathways. Chinese herbal ingredients prefer to regulate the downstream proteins of apoptosis associated pathway; while the western drugs incline to regulate the upstream proteins of VECC (Vascular Epidermal Cells Contraction) related pathways.
\end{abstract}

Conclusion: In summary, the characteristics identified in this study would be valuable for designing new networkbased multi-target CVD drugs or vaccine adjuvants.

\section{Background}

According to the statistics of The World Health Organization (WHO), CVDs are the world's largest killers of human health, since these disorders lead to 17.1 million deaths per year and the death number is still rising [1]. Cardiovascular diseases (CVDs) is the general term that describes a number of circulatory system diseases, which mainly include ischemic heart disease, hypertension, cardiac arrhythmias, stroke, myocardial infarction, coronary artery disease, hypertrophic cardiomyopathy, hyperlipidemia, etc $[2,3]$.

\footnotetext{
* Correspondence: zwcao@tongji.edu.cn; kltang@tongji.edu.cn

'State Key Laboratory of Bioreactor Engineering, East China University of Science \& Technology, Shanghai 200237, China

${ }^{2}$ Shanghai Center for Bioinformation Technology, Shanghai 201203, China
} Full list of author information is available at the end of the article
Both genetic abnormalities and environmental factors play important roles in CVDs development. Their pathological mechanisms often refer to many complex physiological processes, such as inflammation [3], apoptosis [4,5], oxidative stress [6], and lipid metabolism [7].

There have been various anti-CVD drugs approved by US FDA. One of the most-used examples is the statins. They have reduced the mortality and morbidity from atherosclerotic heart disease by about $30 \%$, but the remaining 70\% CVDs still need novel therapies [8]. In recent years, with the development of genomics [9] and proteomics [10], a large number of associated genomic and gene regulation data make it possible to develop new drugs for CVDs. Bezafibrate [11], Lisinopril [12], Quinapril [13] and so on, have been developed as new 
drugs of CVDs. Although western drugs have achieved great successes in CVDs treatment, they may lead to serious side effects [14] and drug resistance [15].

On the other hand, more and more studies found that Chinese herbs have efficient therapeutic effect for CVDs, such as Danshen Pian [16,17], Yin Xing Ye Pian [16,18], Shengmai Yin [16] and Xinning Pian [16]. For instance, Danshen has a range of potentially beneficial effects, including causing coronary vasodilatation, suppressing the formation of thromboxane, and inhibiting platelet adhesion and aggregation [19]. G. biloba extract (GBE) was found to decrease capillary permeability, inhibit platelet-activating factors, and decrease vascular resistance [20]. Herbal ingredients have been expected as a potential drug like database [21]. In addition, increased research has been carried out in search of new adjuvant candidates from traditional Chinese medicinal herbs [22,23]. Although great promise has been shown for Chinese herbs, their therapeutic mechanism is still not very clear.

Before demystify the mechanism of anti-CVD Chinese herbs, researchers want to know whether there is any difference between herbal ingredients and western drugs? It may be a good view towards molecules and their targets to decipher these questions. In this study, herbal ingredients (active compounds in herb which have been reported with anti-CVD effects) and western drugs (FDAapproved drugs with anti-CVD effectiveness) have been comprehensively collected. Their corresponding target proteins were retrieved from DrugBank [24] and HIT [25]. Then, the similarities and the differences of molecular mechanism between those herbal ingredients and western drugs were probed from 3 target-based perspectives: biochemical property, regulated pathway, and disease related network.

\section{Methods and materials \\ Data preparation \\ Molecules, targets and related diseases}

Western drugs whose first letter of ATC code is "C" and their associated targets were downloaded from DrugBank. The keywords such as: "Hypertension", "Hyperlipidemia", "Coronary heart disease", "Stroke", "Myocardial infarction", "Angina", "Atherosclerosis", "Cardiomyopathy", "Heart failure" and "Thrombosis" have been used to search anti-CVD herbs in Chinese Pharmacopoeia [16]. The ingredients of anti-CVD herbs and their corresponding targets were extracted from HIT [25]. Two kinds of ingredients' targets have been collected from HIT: direct targets and indirect targets.

Targets and associated diseases were collected from Therapeutic Target Database (TTD) [26]. As targets are under different stages of study, they have been classified into three categories in TTD: Successful targets, Clinical trial targets and Research targets.

\section{Protein biochemical family, structure domain and cell location}

Protein Biochemical family data was downloaded from UniProtKB [27]. Protein structure domain data was obtained from Pfam [28]. Protein cell location data was collected from The Gene Ontology (GO) [29]. GO provides an ontology of defined terms representing gene product properties, cell location information for the targets was extracted from Cellular component item.

\section{Protein transcription factor}

TRANSFAC database records eukaryotic transcription regulating DNA sequence elements and the transcription factors binding to and acting through them [30]. Information of transcription factor was retrieved from TRANSFAC, 618 transcription factors have been included in TRANSFAC.

\section{Network construction and analysis Construction of target-pathway network and compound- pathway network}

Firstly, target proteins of herbal ingredients and western drugs were mapped onto KEGG pathways [31] respectively. Targets were considered to participate in a specific pathway if they appear in the pathway. Through regulating those targets, western drugs or herbal ingredients act on pathways. A bipartite graph was constructed by linked pathways and targets, which represents the association of targets and pathways [32]. In the same way, the compound (a compound means an herbal ingredient or a western drug)-pathway network was generated.

\section{Degree distribution of network}

In network, the number of edges linked to a node was defined as degree. The degree distribution $f(x)$ of network is the frequency of nodes with degree $\mathrm{x}$. It is reported that degree distribution of the majority of real world networks especially biological networks obey power law. In another word, the majority of nodes in network would only influence few nodes, while few nodes would affect a lot of nodes and play important roles in whole network [32,33].

\section{Pathway enrichment for targets of herbal ingredients and}

\section{western drugs}

Pathway enrichment analysis [34] has been applied to query whether a compound regulate a pathway by chance or not. Fisher's exact test was used. A significance level with a Pvalue less than 0.01 means the compound would regulate the pathway at a high probability [32].

\section{Construction of disease network}

In previous reported studies, inflammation, apoptosis and $\mathrm{Ca}^{2}{ }^{+}$channel caused vascular epidermal cells contraction disorder were the main pathogenesis for cardiovascular diseases. Firstly, two CVDs local networks were constructed: the CVDs-Apoptosis-Network and the CVDsVECC (vascular epidermal cells contraction)-Network. Secondly, the whole-CVDs-network was built which 
integrates CVDs associated pathways in KEGG, BioCarta [35] and Therapeutically Relevant Multiple Pathways (TRMP) [36].

\section{Results and discussions}

Targets of herbal ingredients and western drugs

184 FDA-approved anti-CVD western drugs as well as their 204 protein targets were collected from DrugBank. 40 anti-CVD herbs were collected in the Chinese Pharmacopoeia as well. For these anti-CVD herbs, 172 herbal ingredients and 862 protein targets were identified from the HIT, among which 118 were direct targets.

Targets of herbal ingredients and western drugs were mapped to TTD [37] (Table 1). Targets in TTD have been mainly grouped into three categories: Successful targets, Clinical trial targets and Research targets. Among the collected western drug's targets, 81(39.7\%) are successful targets, $15(7.4 \%)$ are clinical trial targets, $29(14.2 \%)$ are research targets and $2(1.7 \%)$ are discontinued targets. For the direct targets of herbal ingredients, $32(27.1 \%)$ are successful targets, $10(8.5 \%)$ are clinical trial targets, 32(27.1\%) are research targets and 3(2.5\%) are discontinued targets. Additionally, among all targets (including direct and indirect targets) of herbal ingredients, $84(9.7 \%)$ are successful targets, $88(10.2 \%)$ are clinical trial targets, $180(20.9 \%)$ are research targets and 14 (1.6\%) are discontinued targets. Totally, $62.3 \%$ targets of western drugs, $65.3 \%$ of direct targets herbal ingredients and $41.5 \%$ all targets of herbal ingredients are recorded in TTD. Herbal ingredients' direct targets were more similar to western drugs' targets than all targets of herbal ingredients. To better compare herbal ingredients and western drugs, the bias of their target number and target type should be excluded. Only direct targets of herbal ingredients were used in the following study.

$D / t$ values (the average value for the number of associated diseases for each target) were calculated for herbal ingredients' and western drugs' targets (Table 2). Herbal ingredients showed higher $d / t$ value than western drugs in each category. The average number of herbal ingredients' targets associated diseases is higher than that of western ones. It implies that herbal ingredients' targets may associate with more diseases than the targets of western drugs.
To some extent, the number of targets associateddiseases depends on the complexity of the biological function of the target. Since the domain types of protein targets were often used to measure the biological function complexity, distribution of the number of structure domain for targets of western drugs and herbal ingredients were obtained by Pfam (Figure 1a). The numbers of structure domain of targets from herbal ingredients tends to be more than those of western drugs. It indicates that the biological function of the herbal ingredients' targets is more complex than that of the western drugs. This is also consistent with the observation from the $d / t$ value analysis. In another word, herbal ingredients' targets would link to more diseases and prefer to participate in more complicated biological functions.

As shown in Additional file 1, figure S1, 27\% of western drugs bind different subtypes of the same type of protein targets such as inhibitors of calcium ion channel etc. The other $28 \%$ of western drugs regulate different targets in the same biological process. That is to say, besides onetarget-drugs, most targets of multi-targets western drugs either belong to the same protein type or participate in the same biological process. This might hint why western drugs are often used for a certain disorder.

Protein targets were classified into certain families: enzymes, channels and transporters, receptors, factors and regulators, structure proteins, binding proteins and others [38]. As seen from Figure 1b, targets of herbal ingredients were enriched in enzymes, factors and regulators. Western drugs' targets were enriched in receptors, channels and transporters. To some extent, the biochemical family of proteins depends on the cellular location of the proteins. The cellular locations of targets from herbal ingredients and western drugs were extracted from GO (Figure 1c). Targets of herbal ingredients preferred to locate in cytoplasm and nucleus, while western drugs preferred to locate in plasma membrane. As we know, enzymes, factors and regulators often locate in cytoplasm and nucleus, channels and transporters, receptors usually locate in plasma membrane. The results in cellular location accorded with the bio-chemical family analysis. Therefore, herbal ingredients may prefer to target

Table 1 Protein targets of herbal ingredients and western drugs in TTD.

\begin{tabular}{lllllll}
\hline Class & \multicolumn{2}{l}{ western drugs-targets(204) } & \multicolumn{2}{l}{ herbal ingredients -direct targets(118) } & \multicolumn{2}{l}{ herbal ingredients -all targets(862) } \\
\cline { 2 - 7 } & number of targets & Percentage & number of targets & Percentage & number of targets & Percentage \\
\hline Successful targets & 81 & $39.7 \%$ & 32 & $27.1 \%$ & 84 & $9.7 \%$ \\
Clinical trial targets & 15 & $7.4 \%$ & 10 & $8.5 \%$ & 88 & $10.2 \%$ \\
Research targets & 29 & $14.2 \%$ & 32 & $37.1 \%$ & 180 & $20.9 \%$ \\
Discontinued targets & 2 & $1.7 \%$ & 3 & $2.5 \%$ & 14 & $1.6 \%$ \\
Sum of targets in TTD & 127 & $62.3 \%$ & 77 & $65.3 \%$ & 358 & $41.5 \%$ \\
\hline
\end{tabular}


Table 2 Drug targets related diseases from TTD.

\begin{tabular}{llll}
\hline Type of targets & & Number of diseases & $\mathbf{d} / \mathbf{t}$ \\
\hline \multirow{4}{*}{ western drugs } & Successful target(81) & 221 & 2.73 \\
& Clinical trial target(15) & 45 & 3.0 \\
& Research target(29) & 56 & 1.93 \\
& Successful target(32) & 160 & 5.0 \\
herbal ingredients & Clinical trial target(10) & 39 & 3.9 \\
& Research target(32) & 76 & 2.38 \\
\hline
\end{tabular}

$(\mathrm{d} / \mathrm{t}$ represents the average value for the number of associated diseases for each target)

enzymes, factors and regulators in cytoplasm and nucleus, while western drugs may prefer to target channels and transporters, receptors in plasma membrane.

11 targets of 118 herbal ingredients' direct targets were TFs (transcript factor), while only 10 out of 204 western drugs targets were TFs (Table 3). Fisher's exact test was used to quantitatively measure which kind of targets is more enriched with TFs. The P-value of herbal ingredients is less than 0.01 , however western drugs' is greater than 0.05. It implies that herbal ingredients prefer to target TFs than western drugs.

As summarized from the above protein family analysis, cellular location analysis, and the TF enrichment analysis, the targets of western drugs are enriched in channels and transporters, receptors, and they tend to locate on the plasma membrane, which agrees with the common sense about western drug discovery. On the other hand, the targets of herbal ingredients are enriched in enzymes, factors and regulators, and they prefer to locate in cytoplasm and nucleus. This suggests that herbal ingredients may provide new insights for CVDs therapy. In addition, as the herbal ingredients' targets locate in cytoplasm and nucleus, this may meet difficulties in exploring herbal ingredients' mechanism.

\section{Pathways analysis}

To get an overall view of the interplays between herbal ingredients/western drugs and KEGG PATHWAY, we mapped all the targets of herbal ingredients and western

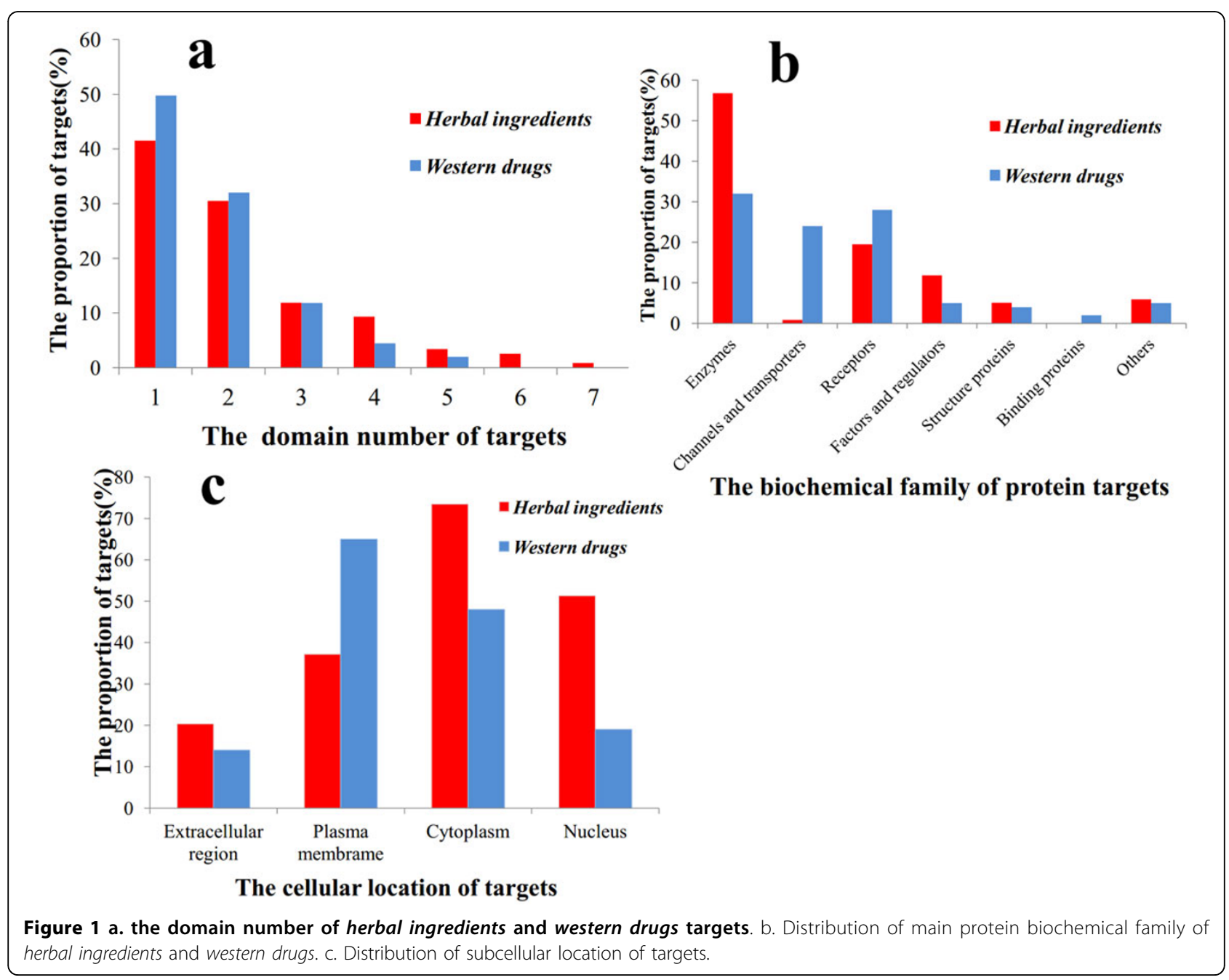


Table 3 Transcription factor distribution for targets of western drug and herbal ingredients

\begin{tabular}{llll}
\hline & Uniprot-id & Factor name & $\begin{array}{l}\text { Num for Transcriptional } \\
\text { genes }\end{array}$ \\
\hline \multirow{6}{*}{ Western drugs } & P10275 & AR & 3 \\
& P05412 & C-Jun & 19 \\
& P0150 & GR-alpha & 5 \\
& P10827 & MR & 0 \\
& P10828 & T3R-alpha & 4 \\
& P35869 & AhR & 5 \\
& Q07869 & PPAR-alpha & 7 \\
& Q03181 & PPAR-beta & 1 \\
& P51843 & DAX1 & 0 \\
P05412 & C-Jun & 19 \\
P03372 & ER-alpha & 8 \\
Q04206 & RelA & 12 \\
Q00613 & HSF1 (long/low) & 1 \\
ingredients & Q09472 & P300 & 15 \\
& P40763 & STAT3 & 3 \\
P35869 & AhR & 7 \\
Q07869 & PPAR-alpha & 0 \\
Q03181 & PPAR-beta & 1 \\
O75469 & PXR-1/SXR & 0 \\
Q92731 & ER-beta & 1 \\
\hline
\end{tabular}

(Fisher's exact test P-value, herbal ingredients: 0.00108, Western drug: 0.14684)

drugs onto the KEGG PATHWAY [31] respectively. It is found that they are enriched in 65(herbal ingredients) and 35 (western drugs) pathways (P-value $<0.01$ ). However, no matter for herbal ingredients' or western drugs targets, at least 33\% pathways of the enriched pathways was Disease pathway (Additional file 2, figure S2). We next mapped herbal ingredients and western drugs targets onto the basic KEGG PATHWAY (without the disease pathway in KEGG PATHWAY) respectively and found that they enriched in 35(herbal ingredients) and 25(western drugs) basic KEGG pathways. Target-pathway network of herbal ingredients and western drugs were illustrated in Figure 2, 3. In herbal ingredients target-pathway network, signal transduction pathways and immune system associated signaling pathways, apoptosis associated pathways interacted with quite plenty of targets, and these pathways also showed high significance enrichment. In western drugs target-pathway network, cardiac and vascular smooth muscle contraction associated pathways interacted with many targets.

Compound-pathway network was constructed, where a compound and a pathway were linked each other if one target of the compound was included in that pathway (Figure 4,5). The signal transduction and immune system associated signaling pathway, apoptosis associated pathways have a higher degree than others in herbal ingredient-pathway network. Vascular smooth muscle contraction associated pathways showed high degree in drug-pathway network for western drugs.

It can be inferred from both target-pathway and compound-pathway analysis that signal transduction and immune system associated signaling pathways, apoptosis associated pathways are always the most important pathway of herbal ingredients, while for the western drugs, the most important pathways are cardiac and vascular smooth muscle contraction associated pathways. These herbal ingredients can be exerted along with the vaccine to elicit a faster and stronger immune response [22].

\section{Disease network}

In order to further pathway analysis, we constructed CVDs-Apoptosis-Network and CVDs-VECC (Vascular Epidermal Cells Contraction)-Network and CVDs-WholeNetwork. In previous studies, cellular apoptosis plays an important role in the development of some CVDs [39], inhibiting apoptosis was an effective way to prevent and control heart failure [5]. The CVDs-Apoptosis-Network was constructed (Figure 6) by integrating known apoptosis associated pathways in the process of CVDs development. There were many targets of herbal ingredients participated in the network, but only two targets of western drugs acted on it. The targets participated in CVDs-Apoptosis-Network further indicate that herbal ingredients preferred to cure CVDs through regulating cellular apoptosis.

Vascular Endothelial Cell Contraction (VECC) was one of the factors for the development of some CVDs, and some drugs had been reported to cure hypertension by regulating vascular endothelial cell contraction associated pathways [40]. Thus, a CVDs-VECC-Network (Figure 7) was constructed by integrating VECC associated pathways in the process of CVDs development. Many of the targets of western drugs present in the network, while only two targets of herbal ingredients presented. This agreed with the hint from former pathway analysis that western drugs preferred to target cardiac and vascular smooth muscle contraction associated pathways.

The CVDs-Whole-Network was constructed by integrating CVDs associated pathways included in KEGG and Therapeutically Relevant Multiple Pathways (TRMP) Database (Figure 8). Western drugs trend to target blood proteins and membrane proteins, while herbal ingredients only targeted one blood protein and most of its targets located inside the cell.

The CVDs-Whole-Network and the two local networks provide an overview that western drugs prefer to target the upstream of CVDs, while the herbal ingredients prefer to modulate the downstream of CVDs.

\section{Adjuvant discussion}

Use of adjuvant in the vaccine preparation is a long standing practice. Despite major advances in vaccine adjuvants, 


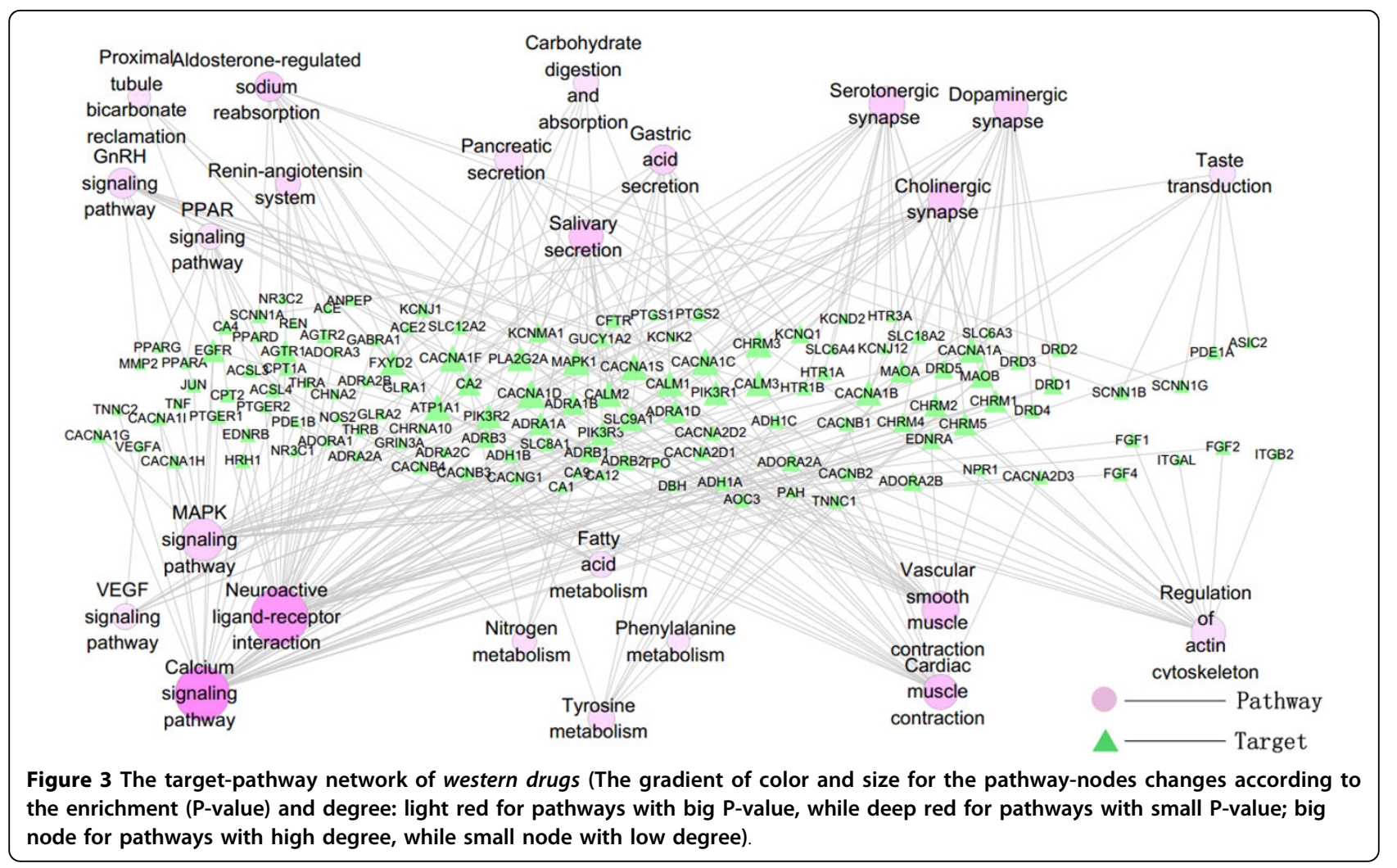



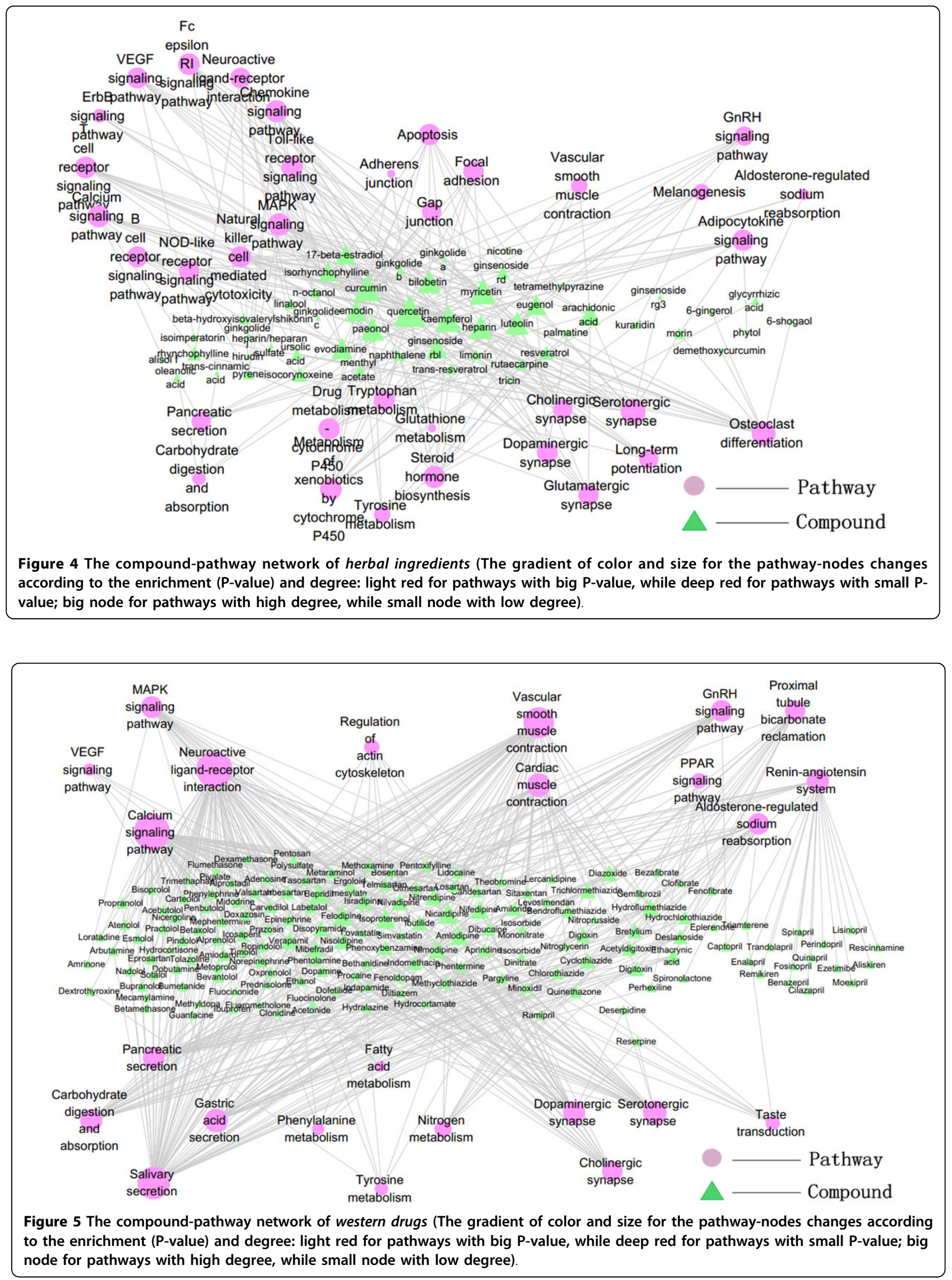


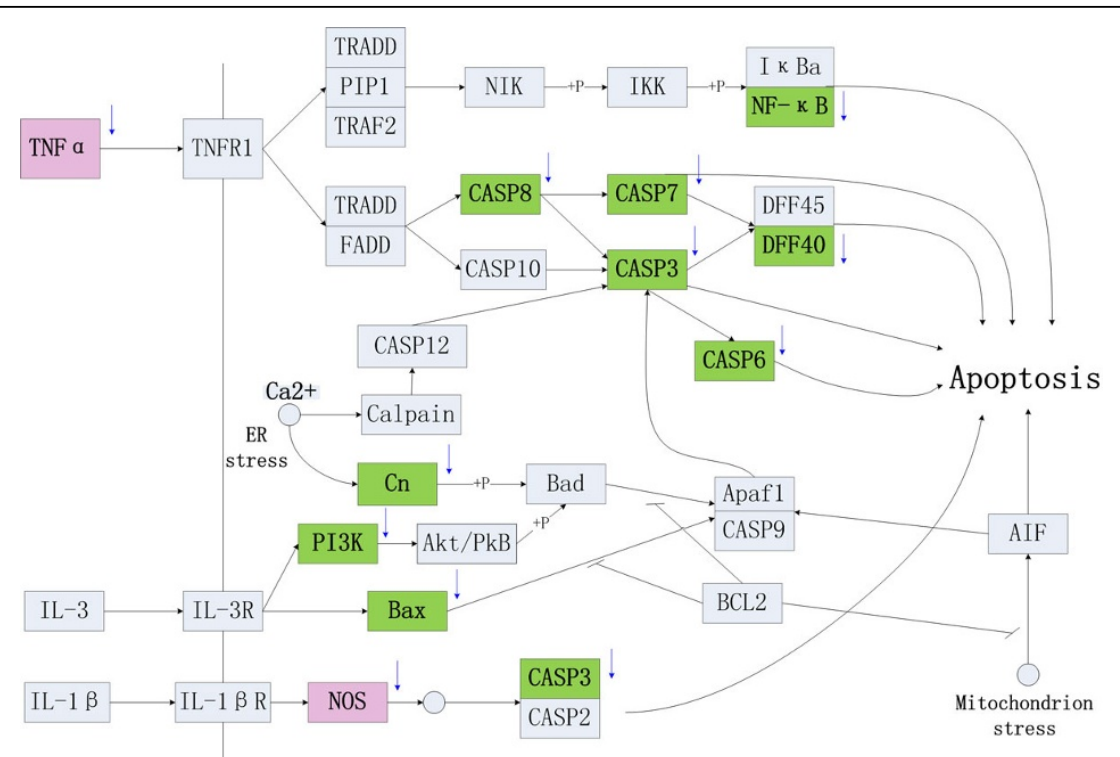

Figure 6 The CVDs-Apoptosis-Network (Different colors represent Different type of targets: the green is the targets of herbal ingredients, and the light red is common targets for western drugs and herbal ingredients. The arrow near the rectangle represents the activities of compounds for the protein: up means activation, down means inhibition).

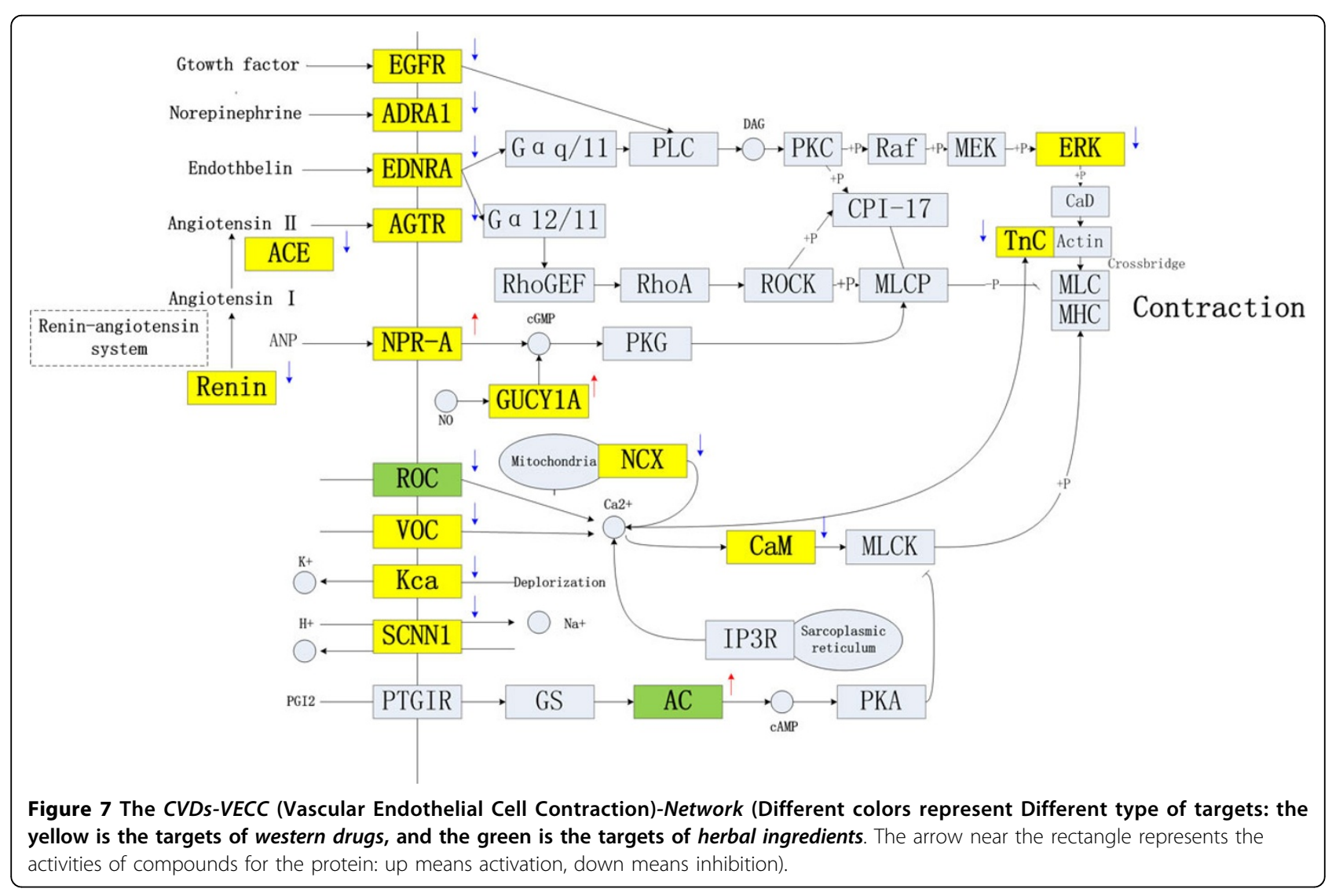




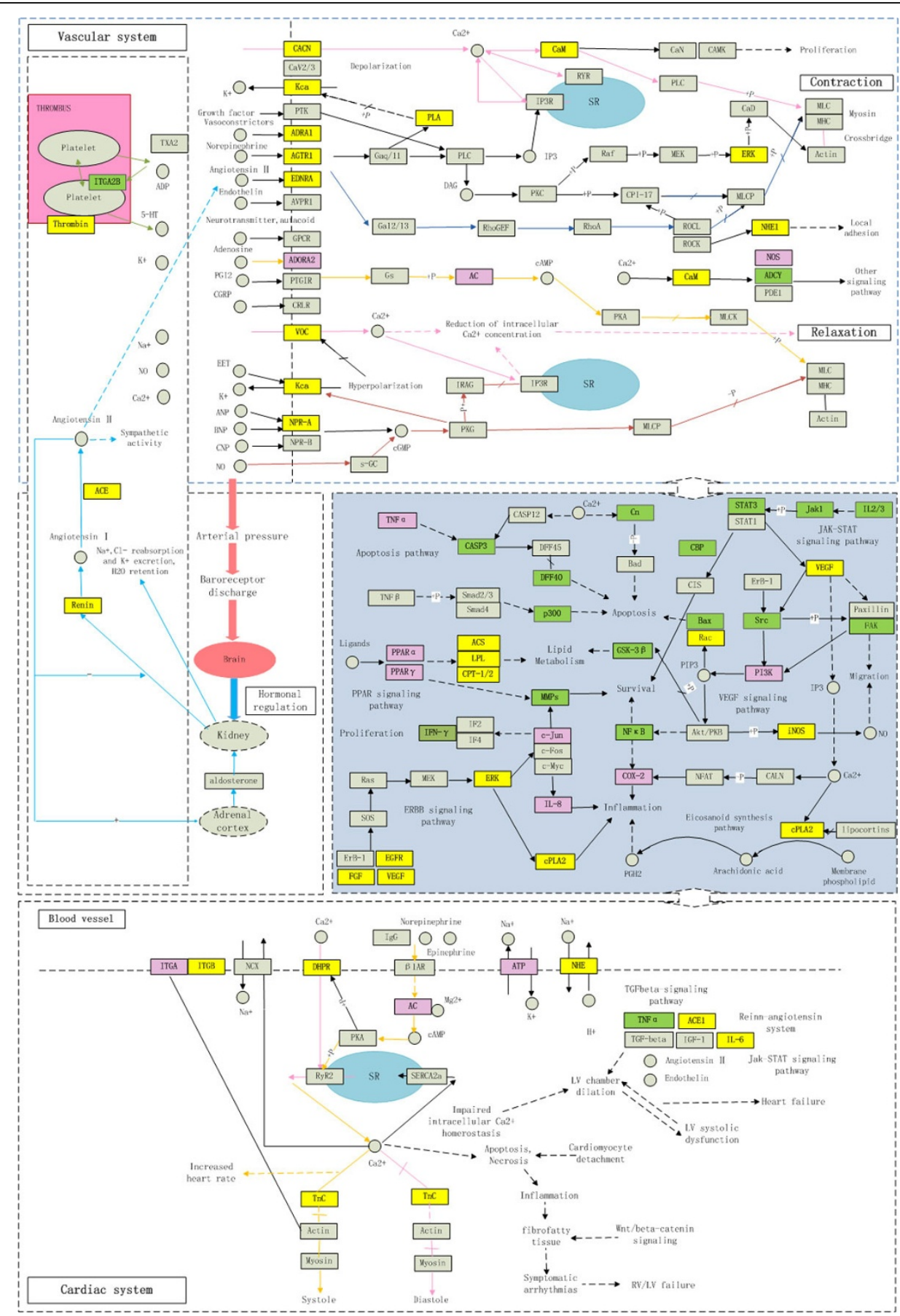

Figure 8 The CVDs-Whole-Network (Different colors represent Different type of targets: the yellow is the targets of western drugs, the green is the targets of herbal ingredients, and the light red is common targets of western drugs and herbal ingredients. The arrow near the rectangle represents the activities of drugs or herbal ingredients for the protein: up means activation, down means inhibition).

vaccines seem to depend on aluminium salts. However, these adjuvants would lead to serious adverse effects [41]. Herbal ingredients as immuno-modulator are paving its way as a safe alternative.

From the results of pathway enrichment, herbal ingredients' direct target significantly enriched in seven immune system pathways, while no immune system pathway was enriched by western drugs' targets. For example, curcumin (the extractive of radix curcumae) would not only regulate interleukin 1(IL1), interleukin 6(IL6), interleukin 8(IL8), interferon $-\gamma($ IFN $-\gamma)$ and Transforming Growth Factor beta (TGF- $\beta$ ) in T and B cell, but also target some immune 
proteins such as, Toll-like receptor 4(TLR4), CD80 and CD28. Styrene (the extractive of storax) can affect IL4, IL5, IL13 and TGF- $\beta$ expression. Resveratrol (the extractive of polygonum cuspidatum) can regulate IL1, IL6, IL8, IL10, IFN- $\gamma$, TGF- $\beta$, CD80 and CD28. What's more, ginseng saponins have adjuvant effects on the specific immune responses, which due to their can promote IL1 production by pertoneal macrophages, stimulate secretion of cytokine such as IL2, IL4, IL6, IL8, IL10, IFN- $\gamma$ and TNF- $\alpha$, regulate TLR4 to produce proinflammatory cytokines, and affect TGF- $\beta$ to enhance lymphocyte proliferation [42-45]. Therefore, those herbal ingredients could regulate one or several kinds of cytokine such as IL1, IL4, IL6, IL8, IL10, IFN- $\gamma$, TGF- $\beta$ and immune related protein TLR4 have the potential to promote both humoral and cellular immune responses. Some ingredients of anti-CVD herbs might be the candidate adjuvant.

\section{Conclusion}

Herbal ingredients and western drugs, as well as their targets were thoroughly collected in this work. The similarities and the differences between herbal ingredients and western drugs were deeply explored. From biochemical property analysis, the biological function of herbal ingredients' targets is more complex than that of the targets of western drugs. Herbal ingredients tend to target enzymes, factors, regu-lators protein in cytoplasm and nucleus, while western drugs lean towards targeting the protein of channels and transporters, receptors in plasma membrane. From pathway analysis, the signal transduction and immune system associated signaling pathways, apoptosis associated pathways are always the most important pathway for herbal ingredients, however western drugs incline to regulate vascular smooth muscle contraction associated pathways. From disease network analysis, herbal ingredients prefer to regulate the downstream proteins of apoptosis associated pathway, while the western drugs incline to regulate the upstream proteins of VECC related pathways.

According to some statistics, nearly one-third of the topselling drugs in the world are nature products or their derivatives, and nature products are the most consistently successful source of drug leads $[46,47]$. Herbal ingredients may provide some new clues to drugs development for CVDs. Herbal ingredients may also have the potential to be used in design of new vaccines.

\section{Additional material}

Additional file 1: Figure S1 The distribution of drug type for western drugs.

Additional file 2: Figure S2 The proportional distribution of enriched KEGG pathway.
Competing interests

The authors declare that they have no competing interests.

\section{Authors' contributions}

PF and LY collected data and performed the computational data analysis. ZWC and KLT conceived of the study, participated in its design and coordination and revised the manuscript. LLY and YS participated in the design and helped to review the manuscript. All authors read and approved the final manuscript.

\section{Acknowledgements}

The work was partly supported by Ministry of Science and Technology China (2012AA020404), and National Natural Science Foundation of China (30900832). The publication of paper was funded by grants from Ministry of Health (2012ZX10005001-008)

\section{Declarations}

The publication of this work was funded by Ministry of Health (2012ZX10005001-008)

This article has been published as part of BMC Bioinformatics Volume 15 Supplement 4, 2014: Selected articles on Computational Vaccinology 2013. The full contents of the supplement are available online at http://www. biomedcentral.com/bmcbioinformatics/supplements/15/S4.

\section{Authors' details}

${ }^{1}$ State Key Laboratory of Bioreactor Engineering, East China University of Science \& Technology, Shanghai 200237, China. ${ }^{2}$ Shanghai Center for Bioinformation Technology, Shanghai 201203, China. ${ }^{3}$ School of Life Science and Technology, Tongji University, Shanghai 200092, China.

Published: 19 March 2014

\section{References}

1. Cartwright EJ, Oceandy D, Austin C, Neyses L: Ca2+ signalling in cardiovascular disease: the role of the plasma membrane calcium pumps. Sci China Life Sci 2011, 54(8):691-698.

2. Nabel EG: Cardiovascular disease. N Engl J Med 2003, 349(1):60-72.

3. Hansson GK, Robertson AK, Soderberg-Naucler C: Inflammation and atherosclerosis. Annu Rev Pathol 2006, 1:297-329.

4. Singh SS, Kang PM: Mechanisms and inhibitors of apoptosis in cardiovascular diseases. Curr Pharm Des 2011, 17(18):1783-1793.

5. Kim GT, Chun YS, Park JW, Kim MS: Role of apoptosis-inducing factor in myocardial cell death by ischemia-reperfusion. Biochem Biophys Res Commun 2003, 309(3):619-624.

6. Keith M, Geranmayegan A, Sole MJ, Kurian R, Robinson A, Omran AS, Jeejeebhoy KN: Increased oxidative stress in patients with congestive heart failure. J Am Coll Cardiol 1998, 31(6):1352-1356.

7. Cheng TJ, Chuu JJ, Chang CY, Tsai WC, Chen KJ, Guo HR: Atherosclerosis induced by arsenic in drinking water in rats through altering lipid metabolism. Toxicol Appl Pharmacol 2011, 256(2):146-153.

8. Plump AS, Lum PY: Genomics and cardiovascular drug development. J Am Coll Cardiol 2009, 53(13):1089-1100.

9. Frazier L, Johnson RL, Sparks E: Genomics and cardiovascular disease. J Nurs Scholarsh 2005, 37(4):315-321.

10. Mayr M, Zhang J, Greene AS, Gutterman D, Perloff J, Ping P: Proteomicsbased development of biomarkers in cardiovascular disease: mechanistic, clinical, and therapeutic insights. Mol Cell Proteomics 2006, 5(10):1853-1864.

11. Tenenbaum A, Fisman EZ, Boyko V, Benderly M, Tanne D, Haim M, Matas Z, Motro M, Behar S: Attenuation of progression of insulin resistance in patients with coronary artery disease by bezafibrate. Arch Intern Med 2006, 166(7):737-741.

12. Nielsen SE, Sugaya T, Tarnow L, Lajer M, Schjoedt KJ, Astrup AS, Baba T, Parving HH, Rossing P: Tubular and glomerular injury in diabetes and the impact of ACE inhibition. Diabetes Care 2009, 32(9):1684-1688.

13. Voors AA, van Geel PP, Oosterga M, Buikema $H$, van Veldhuisen DJ, van Gilst WH: Vascular effects of quinapril completely depend on ACE insertion/deletion polymorphism. J Renin Angiotensin Aldosterone Syst 2004, 5(3):130-134. 
14. Mukhtar RY, Reckless JP: Statin-induced myositis: a commonly encountered or rare side effect? Curr Opin Lipidol 2005, 16(6):640-647.

15. Lau WC, Gurbel PA: Antiplatelet drug resistance and drug-drug interactions: Role of cytochrome P450 3A4. Pharm Res 2006, 23(12):2691-2708.

16. PRC SPCot: Pharmacopoeia of People's Republic of China, 2005. Beijing: Chemical Industry Press; 2005.

17. Li $X, X u X$, Wang J, Yu H, Wang $X$, Yang $H, X u$, Tang S, Li Y, Yang L, Huang $L$, Wang $Y$, Yang $S$ : A system-level investigation into the mechanisms of Chinese Traditional Medicine: Compound Danshen Formula for cardiovascular disease treatment. PLoS One 2012, 7(9):e43918.

18. Lim S, Yoon JW, Kang SM, Choi SH, Cho BJ, Kim M, Park HS, Cho HJ, Shin H, Kim YB, Kim HS, Jang HC, Park KS: EGb761, a Ginkgo biloba extract, is effective against atherosclerosis in vitro, and in a rat model of type 2 diabetes. PLoS One 2011, 6(6):e20301.

19. Cheng TO: Cardiovascular effects of Danshen. Int J Cardiol 2007, 121(1):9-22

20. Kudolo GB, Wang W, Barrientos J, Elrod R, Blodgett J: The ingestion of Ginkgo biloba extract (EGb 761) inhibits arachidonic acid-mediated platelet aggregation and thromboxane B2 production in healthy volunteers. J Herb Pharmacother 2004, 4(4):13-26.

21. Newman DJ, Cragg GM: Natural products as sources of new drugs over the 30 years from 1981 to 2010. J Nat Prod 2012, 75(3):311-335.

22. Sakure S, Negi VD, Mitra SK, Nandakumar KS, Chakravortty D: Vaccine with herbal adjuvant-a better cocktail to combat the infection. Vaccine 2008 26(27-28):3387-3388.

23. Song $X, H u$ S: Adjuvant activities of saponins from traditional Chinese medicinal herbs. Vaccine 2009, 27(36):4883-4890.

24. Wishart DS, Knox C, Guo AC, Cheng D, Shrivastava S, Tzur D, Gautam B, Hassanali M: DrugBank: a knowledgebase for drugs, drug actions and drug targets. Nucleic Acids Res 2008, 36(Database):D901-906.

25. Ye H, Ye L, Kang H, Zhang D, Tao L, Tang K, Liu X, Zhu R, Liu Q, Chen YZ, Li Y, Cao Z: HIT: linking herbal active ingredients to targets. Nucleic Acids Res 2011, 39(Database):D1055-1059.

26. Zhu F, Shi Z, Qin C, Tao L, Liu X, Xu F, Zhang L, Song Y, Zhang J, Han B, Zhang $P$, Chen Y: Therapeutic target database update 2012: a resource for facilitating target-oriented drug discovery. Nucleic Acids Res 2012, 40(Database):D1128-1136.

27. Magrane $\mathrm{M}$, Consortium U: UniProt Knowledgebase: a hub of integrated protein data. Database (Oxford) 2011, 2011:bar009.

28. Punta M, Coggill PC, Eberhardt RY, Mistry J, Tate J, Boursnell C, Pang N, Forslund K, Ceric G, Clements J, Heger A, Holm L, Sonnhammer EL, Eddy SR, Bateman A, Finn RD: The Pfam protein families database. Nucleic Acids Res 2012, 40(Database):D290-301.

29. Gene Ontology Database. [http://www.geneontology.org/]

30. Wingender $E$, Dietze $P$, Karas $H$, Knuppel R: TRANSFAC: a database on transcription factors and their DNA binding sites. Nucleic Acids Res 1996, 24(1):238-241

31. Ogata H, Goto S, Sato K, Fujibuchi W, Bono H, Kanehisa M: KEGG: Kyoto Encyclopedia of Genes and Genomes. Nucleic Acids Res 1999, 27(1):29-34

32. Zhao J, Yang P, Li F, Tao L, Ding H, Rui Y, Cao Z, Zhang W: Therapeutic effects of astragaloside IV on myocardial injuries: multi-target identification and network analysis. PLOS One 2012, 7(9):e44938.

33. Barabasi AL, Albert R: Emergence of scaling in random networks. Science 1999, 286(5439):509-512.

34. Curtis RK, Oresic M, Vidal-Puig A: Pathways to the analysis of microarray data. Trends Biotechnol 2005, 23(8):429-435.

35. BioCarta Database. [http://www.biocarta.com/].

36. Zheng CJ, Zhou H, Xie B, Han LY, Yap CW, Chen YZ: TRMP: a database of therapeutically relevant multiple pathways. Bioinformatics 2004, 20(14):2236-2241.

37. Gonzalez-Gay MA, Gonzalez-Juanatey C: Inflammation, endothelial function and atherosclerosis in rheumatoid arthritis. Arthritis Res Ther 2012, 14(4):122.

38. Drews J: Drug discovery: a historical perspective. Science 2000 287(5460):1960-1964.

39. Kim NH, Kang PM: Apoptosis in cardiovascular diseases: mechanism and clinical implications. Korean Circ J 2010, 40(7):299-305.

40. Seok YM, Jin F, Shin HM, Sung SH, Sohn UD, Cho JY, Kim IK: HMC05 attenuates vascular contraction through inhibition of RhoA/Rho-kinase signaling pathway. J Ethnopharmacol 2011, 133(2):484-489.
41. Shaw CA, Petrik MS: Aluminum hydroxide injections lead to motor deficits and motor neuron degeneration. J Inorg Biochem 2009, 103(11):1555-1562

42. Rivera E, Ekholm Pettersson F, Inganas M, Paulie S, Gronvik KO: The Rb1 fraction of ginseng elicits a balanced Th1 and Th2 immune response. Vaccine 2005, 23(46-47):5411-5419.

43. Yesilada E, Bedir E, Calis I, Takaishi Y, Ohmoto Y: Effects of triterpene saponins from Astragalus species on in vitro cytokine release. J Ethnopharmacol 2005, 96(1-2):71-77.

44. Sun $H, Y e Y$, Pan $Y$ : Immunological-adjuvant saponins from the roots of Panax notoginseng. Chem Biodivers 2005, 2(4):510-515.

45. Nakaya TA, Kita M, Kuriyama H, Iwakura $Y$, Imanishi J: Panax ginseng induces production of proinflammatory cytokines via toll-like receptor. J Interferon Cytokine Res 2004, 24(2):93-100.

46. Harvey A: Strategies for discovering drugs from previously unexplored natural products. Drug Discov Today 2000, 5(7):294-300.

47. Strohl WR: The role of natural products in a modern drug discovery program. Drug Discov Today 2000, 5(2):39-41.

doi:10.1186/1471-2105-15-S4-S3

Cite this article as: Fu et al:: Target network differences between western drugs and Chinese herbal ingredients in treating cardiovascular disease. BMC Bioinformatics 2014 15(Suppl 4):S3

\section{Submit your next manuscript to BioMed Central and take full advantage of:}

- Convenient online submission

- Thorough peer review

- No space constraints or color figure charges

- Immediate publication on acceptance

- Inclusion in PubMed, CAS, Scopus and Google Scholar

- Research which is freely available for redistribution

Submit your manuscript at www.biomedcentral.com/submit
C Biomed Central 